\title{
CRYSTAL ARTHRITIS \\ Gout attack prevention with a cherry on top
}

Consumption of cherries helps to prevent attacks of gout, a new epidemiological study from the USA has revealed. A common, highly debilitating disease, gout is known to be both positively and negatively influenced by nutritional factors. Indeed, according to many patients, researchers (and producers of cherries and cherry products), these fruit might be beneficial in gout; "however, no study has assessed whether eating cherries lowers the risk of gout attacks," says Yuqing Zhang, of Boston University, USA. "To help address this knowledge gap, we conducted an online case-crossover study to assess a set of putative triggers, including cherry consumption, in relation to risk of recurrent gout attacks."

Zhang and co-worker's Internetbased study enabled 633 patients with a physician diagnosis of gout to be enrolled from across the USA, and real-time information to be obtained, potentially reducing recall bias. The case-crossover design, in which patients act as their own controls, is highly adaptable to examining associations of acute events with triggers, and reduces observational study design challenges such as control selection bias and variable characteristics of participants.

The data Zhang et al. obtained suggest that eating cherries or cherry extract lowers the risk of repeat gout attacks by $35 \%$. This protective effect was independent of other risk factors including gender, genetics, BMI and purine or alcohol intake, and was greater with increasing cherry consumption; 3 servings (of 10-12 cherries each) over 2 days gave optimal benefit. Moreover, "when cherry intake was combined with allopurinol use the odds of recurrent gout attacks decreased by almost 75\%," states Zhang.

The authors suggest that reduction of serum uric acid levels and the antiinflammatory and antioxidant properties of cherry anthocyanins might explain their findings. However, the study design meant that the investigators were unable to comment on the associations between ingesting cherries, uric acid levels, inflammation and the risk of gout attacks.

These promising results indicate that cherries might represent a safe and effective gout therapy for patients not eligible for urate-lowering therapy,

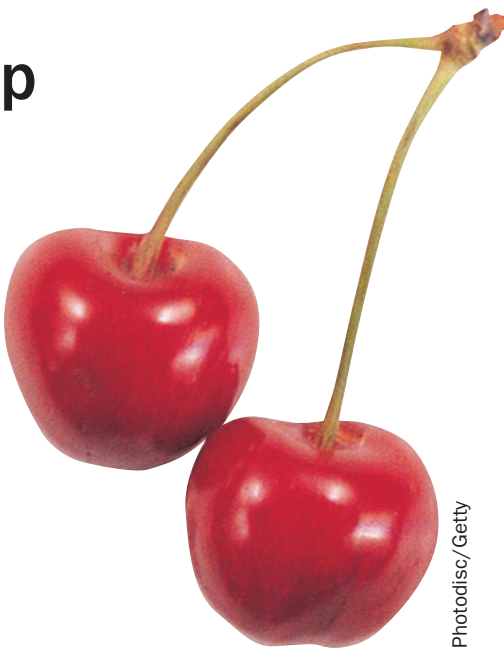

or a useful addition for those who are. However, "until randomized placebocontrolled trials confirm our findings we would not advocate that individuals who suffer from gout abandon standard therapies and opt for cherry extract products as an alternative," concedes Zhang.

David Killock

Original article Zhang, Y. et al. Cherry consumption and the risk of recurrent gout attacks. Arthritis Rheum. doi:10.1002/art.34677 